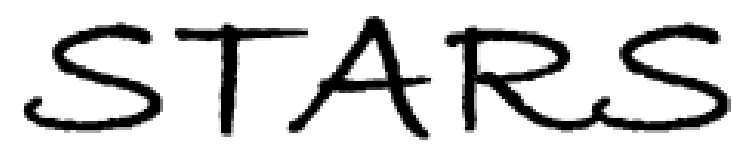

University of Central Florida

STARS

$1-1-2004$

\title{
Polymer translocation through a nanopore induced by adsorption: Monte Carlo simulation of a coarse-grained model
}

Andrey Milchev

Kurt Binder

Aniket Bhattacharya

University of Central Florida

Find similar works at: https://stars.library.ucf.edu/facultybib2000

University of Central Florida Libraries http://library.ucf.edu

This Article is brought to you for free and open access by the Faculty Bibliography at STARS. It has been accepted for inclusion in Faculty Bibliography 2000 s by an authorized administrator of STARS. For more information, please contactSTARS@ucf.edu.

\section{Recommended Citation}

Milchev, Andrey; Binder, Kurt; and Bhattacharya, Aniket, "Polymer translocation through a nanopore induced by adsorption: Monte Carlo simulation of a coarse-grained model" (2004). Faculty Bibliography 2000s. 2585.

https://stars.library.ucf.edu/facultybib2000/2585

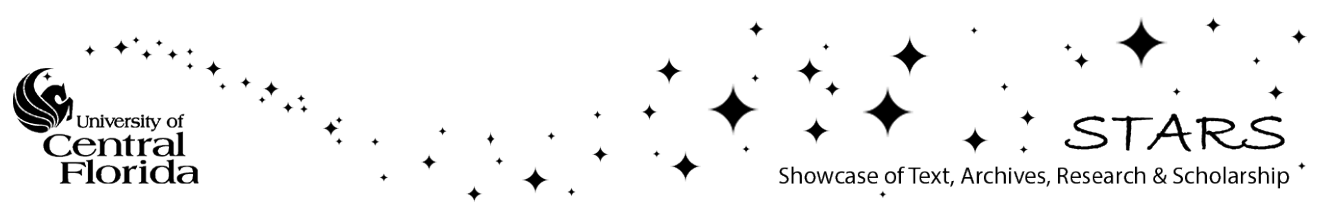




\section{Polymer translocation through a nanopore induced by adsorption: Monte Carlo simulation of a coarse-grained model}

Cite as: J. Chem. Phys. 121, 6042 (2004); https://doi.org/10.1063/1.1785776

Submitted: 15 April 2004 . Accepted: 30 June 2004 . Published Online: 14 September 2004

Andrey Milchev, Kurt Binder, and Aniket Bhattacharya

\section{ARTICLES YOU MAY BE INTERESTED IN}

Polymer translocation through a hole

The Journal of Chemical Physics 111, 10371 (1999); https://doi.org/10.1063/1.480386

Polymer escape through a nanopore

The Journal of Chemical Physics 118, 5174 (2003); https://doi.org/10.1063/1.1553753

Unforced translocation of a polymer chain through a nanopore: The solvent effect

The Journal of Chemical Physics 126, 204901 (2007); https://doi.org/10.1063/1.2735627

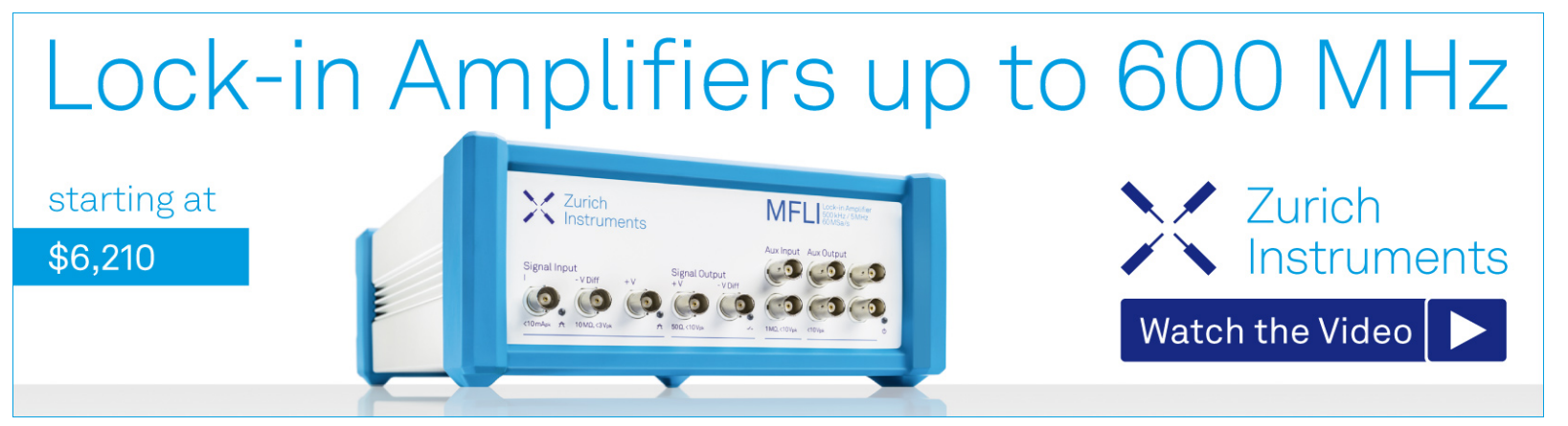

J. Chem. Phys. 121, 6042 (2004); https://doi.org/10.1063/1.1785776

121, 6042

(c) 2004 American Institute of Physics. 


\title{
Polymer translocation through a nanopore induced by adsorption: Monte Carlo simulation of a coarse-grained model
}

\author{
Andrey Milchev ${ }^{\mathrm{a})}$ and Kurt Binder \\ Institut für Physik, Johannes-Gutenberg-Universität Mainz, Staudinger Weg 7, 55099 Mainz, Germany \\ Aniket Bhattacharya \\ Department of Physics, University of Central Florida, Orlando, Florida 32816-2385
}

(Received 15 April 2004; accepted 30 June 2004)

\begin{abstract}
Dynamic Monte Carlo simulation of a bead-spring model of flexible macromolecules threading through a very narrow pore in a very thin rigid membrane are presented, assuming at the cis side of the membrane a purely repulsive monomer-wall interaction, while the trans side is attractive. Two choices of monomer-wall attraction $\varepsilon$ are considered, one choice is slightly below and the other slightly above the "mushroom to pancake" adsorption threshold $\varepsilon_{c}$ for an infinitely long chain. Studying chain lengths $N=32,64,128$, and 256 and varying the number of monomers $N_{\text {trans }}$ (time $t=0)$ that have already passed the pore when the simulation started, over a wide range, we find for $\varepsilon<\varepsilon_{c}$ (nonadsorbing case) that the translocation probability varies proportional to $c_{\text {trans }}$ $=N_{\text {trans }}(t=0) / N$ for small $c_{\text {trans }}$, while for $\varepsilon>\varepsilon_{c}$ a finite number $N_{\text {trans }}(t=0)$ suffices that the translocation probability is close to unity. In the case $\varepsilon<\varepsilon_{c}$, however, the time it takes for those chains to get through the pore to complete the translocation process scales as $\tau \propto N^{2.23 \pm 0.04}$. This result agrees with the suggestion of Chuang, Kantor, and Kardar [Phys. Rev. E 65, 011802 (2001)] that the translocation time is proportional to the Rouse time, that scales under good solvent condition as $\tau_{\text {Rouse }} \propto N^{2 \nu+1}$, with the excluded-volume exponent $\nu \approx 0.59$ in $d=3$ dimensions. Our results hence disagree with the suggestions that the translocation time should scale as either $N^{2}$ or $N^{3}$. For $\varepsilon>\varepsilon_{c}$, we find that the translocation time scales as $\tau \propto N^{1.65 \pm 0.08}$. We suggest a tentative scaling explanation for this result. Also the distribution of translocation times is obtained and discussed. (C) 2004 American Institute of Physics. [DOI: 10.1063/1.1785776]
\end{abstract}

\section{INTRODUCTION}

Translocation of polymers through nanoscopic small holes in membranes recently received great attention in experiment, ${ }^{1-7}$ analytical theory, ${ }^{8-16}$ and simulations. ${ }^{17-23}$ The reason for this interest stems from the fact that the motion of polymers across nanopores is important for many phenomena in biology and for industrial processes. For example, protein transport through channels in biological membranes, motion of DNA molecules across pores into the cell nucleus, virus infection of cells, gene transfer between bacteria are a few examples where polymer translocation plays a role in biological processes. ${ }^{24-26}$ Possible biotechnological applications include DNA migration through microfabricated channels and devices, ${ }^{27,28}$ gene therapy, drug delivery, etc. ${ }^{29}$

The driving mechanisms for this polymer translocation processes have been a subject of intense discussion; e.g., ratchet mechanisms ${ }^{8,18}$ electric fields ${ }^{12,14}$ and chemical potential gradients, ${ }^{9,11,22}$ and selective adsorption on one side of the membrane ${ }^{10}$ were some of the mechanisms discussed in the literature cited above. Since polymer translocation is such an ubiquitous phenomenon, it is not clear that a single universal mechanism is operative under all circumstances, and hence a more detailed study of the various mechanisms is still worth while.

\footnotetext{
a) Also at Institute for Physical Chemistry, Bulgarian Academy of Sciences, 1113 Sofia, Bulgaria.
}

In this spirit, we follow up the suggestion by Park and Sung ${ }^{10}$ that polymer translocation may be induced by adsorption. Park and Sung ${ }^{10}$ considered the limiting case of an extremely long chain, such that both the thickness of the membrane can be taken infinitely thin and the lateral extent of the pore can be taken vanishingly small, in comparison with the linear dimensions of the chain. In addition, Gaussian chain statistics was applied, rendering hence some aspects of the problem analytically solvable, but neglecting the excludedvolume interactions that would be present between the monomers under good solvent conditions. ${ }^{30}$ However, the possibility of reducing the problem to a single degree of freedom passing over a free energy barrier is also controversial. ${ }^{13}$

In the present paper, we shall also consider a coarsegrained bead-spring model of a flexible homopolymer chain; ${ }^{31-36}$ thus, we still ignore both the details of the chemical structure of the biopolymers (as the other simulations ${ }^{17-23}$ did as well) and the chain stiffness. But the model allows implicitly to vary the solvent quality (although the solvent molecules are not explicitly considered) and hence take into account excluded volume as well as attractive interactions among the monomers. ${ }^{31-36}$ In addition, both the nonzero finite radius of the pore and the thickness of the membrane (i.e., the height of the pore) enter as parameters that could be varied in this problem. However, in the present 
first exploratory study only a single choice of these parameters is considered.

The outline of this paper is as follows. In Sec. II, we briefly describe the model and comment on the simulation technique. Section III presents the numerical results. Section IV discusses the interpretation of our findings, and compares them with the work in the literature, ${ }^{9-23}$ while Sec. V contains our conclusions, including a brief outlook to future work.

\section{MODEL AND SIMULATION TECHNIQUE}

For many problems in polymer science, a detailed description of the chemical structure of the polymer is not necessary, and it suffices to focus on a coarse-grained model of a polymer chain that keeps only the essential features of the macromolecule, such as connectivity of the chain molecule, flexibility, and nonbonded (excluded volume) interaction, since no two (effective) monomers sit on top of each other. This is the spirit of the model used here, following previous work $^{31-36}$ where this model was used successfully to study bulk behavior of polymer solutions ${ }^{31-33}$ and the interaction of polymer chains with attractive $e^{34,36}$ or repulsive ${ }^{35}$ walls. Each chain contains $N$ effective monomers (that are thought to comprise several subsequent chemical monomers along the backbone of the chain).

Two successive effective monomers along the chain are connected by an anharmonic spring, described by the finitely extensible nonlinear elastic (FENE) potential

$$
U_{\mathrm{FENE}}=-\frac{K}{2} R^{2} \ln \left[1-\frac{\left(\ell-\ell_{0}\right)^{2}}{R^{2}}\right] .
$$

Here $\ell$ is the length of an effective bond, which can vary in between $\ell_{\min }<\ell<\ell_{\max }$, with $R=\ell_{\max }-\ell_{0}=\ell_{0}-\ell_{\min }, \ell_{0}$ being the equilibrium value for which the potential takes its minimum. Choosing $\ell_{\max } \equiv 1$ as our unit of length and $R$ $=0.3$, we have $\ell_{\min }=0.4, \ell_{0}=0.7$. The spring constant $K$ is taken as $K / k_{B} T=40$. All these choices are identical to our previous work. ${ }^{31-36}$

The nonbonded interactions between the effective monomers are described by the Morse potential ${ }^{32}$

$U_{M}=\varepsilon_{M}\left\{\exp \left[-2 \alpha\left(r-r_{\text {min }}\right)\right]-2 \exp \left[-\alpha\left(r-r_{\text {min }}\right)\right]\right\}$,

where $r$ is the distance between the beads, and the parameters in Eq. (2) are chosen as $r_{\min }=0.8, \varepsilon_{M} \equiv 1$ setting the energy scale, and $\alpha=24$. Owing to the large value of this latter constant, $U_{M}(r)$ decays to zero very rapidly for $r$ $>r_{\text {min }}$, and is completely negligible for distances larger than unity. This choice of parameters is useful from a computational point of view, since it allows the use of a very efficient link-cell algorithm. ${ }^{31}$

Physically this choice of parameters makes sense, recalling that the length $\ell_{0}$ of an effective bond between effective monomers corresponds to a kind of Kuhn segment, and hence is typically at least about $2 \mathrm{~nm}$, rather than the length of a covalent C-C bond (which would only be about $1.5 \AA$ ). Therefore, it is not unreasonable to choose the width of the attractive potential well described by Eq. (2) less than $\ell_{0}$, and also the membrane containing the pore is chosen per-

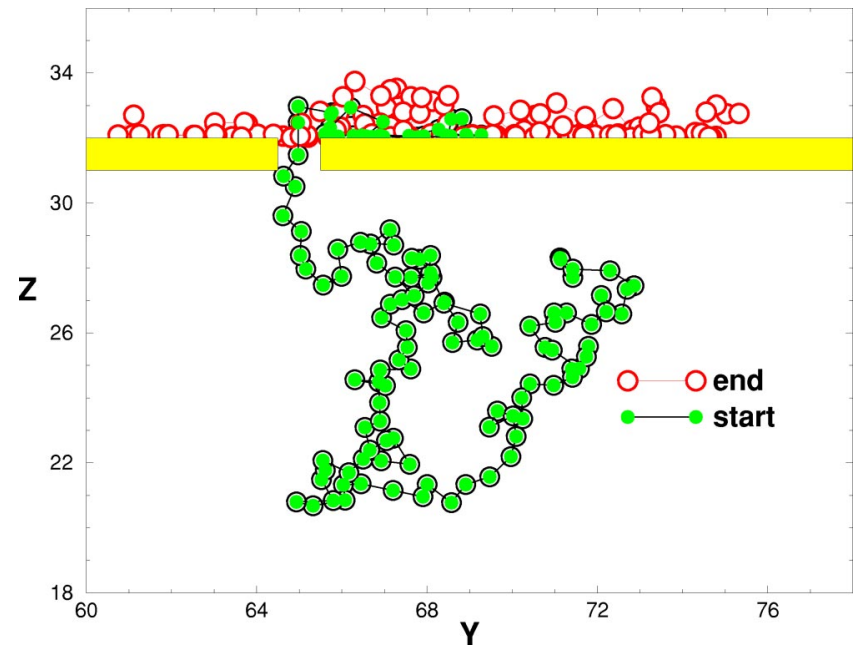

FIG. 1. Two snapshot pictures of polymer chains with $N=128$ at a temperature $T=1$, projected into the $y z$ plane containing the pore (centered at $y$ $=65, z=31.5$ ) in the membrane (which blocks the region from $z=31$ to $z$ $=32$ from occupation by the effective monomers, apart from the volume taken by the pore). The strength of the adsorption potential is $\varepsilon=2.2$. One snapshot refers to the situation that $N_{c i s}=100$ monomers are on the repulsive side of the membrane (marked "start"), the other has all monomers on the attractive side (marked "end"), showing a typical pancake configuration of the polymer.

fectly flat and structureless (in reality it has a roughness on the atomistic scale, but on the scale of $\ell_{0}$ it may be taken effectively smooth). This consideration suggests also that it is physically reasonable to choose both the thickness $d$ of the membrane and the diameter $D$ of the pore in the membrane, through which the polymer chain must move, of the same order as $\ell_{0}$. In the present paper, we hence choose $d \equiv D$ $=1$ throughout. The motion of polymers in much larger pores (where $d \gg 1, D \gg 1$ ) is left to future work (the limiting case $d \rightarrow \infty, D$ finite has already been considered by simulations of related models $\mathrm{s}^{37,38}$ and theoretically ${ }^{39,40}$ ). In order to illustrate the linear dimensions of the simulated chains in relation to our pore geometry, Fig. 1 shows two projections of snapshot pictures of a chain with $N=128$ into the $y z$ plane, which contains the hole; one snapshot has only 28 beads with $z>32$ (i.e., on the adsorbing side of the membrane) while the second snapshot shows the chain fully adsorbed. Note that for simplicity we have assumed a pore of square cross section (compatible with our link-cell scheme) rather than spherical, but we do not expect that such details should matter with respect to the general features of the behavior.

Since for this model in the bulk the $\theta$ temperature has been found ${ }^{33}$ as $k_{B} \Theta \approx 0.62$, all simulations are done for $k_{B} T=1$, to ensure good solvent behavior.

Following Park and Sung, ${ }^{10}$ we consider an asymmetric membrane, one side of which is purely repulsive, while the other side is attractive. The wall potential is chosen as follows, cf. Fig. 1,

$$
\begin{aligned}
& U_{w}^{\text {trans }}(z)=\infty, \quad z<32, \\
& U_{w}^{\text {trans }}(z)=-\varepsilon, \quad 32<z<32+\delta, \quad \delta=1 / 8, \\
& U_{w}^{\text {trans }}(z)=0, \quad z>32+\delta,
\end{aligned}
$$


while on the "cis" side of the membrane the potential is purely repulsive,

$$
\begin{aligned}
& U_{w}^{c i s}(z)=\infty, \quad z>31, \\
& U_{w}^{c i s}(z)=0, \quad z<31 .
\end{aligned}
$$

Note that the size of the total simulation box is chosen so large that a chain interacting with the membrane (Fig. 1) never touches any of the walls confining the box.

Since the dynamics of the polymer translocation through the pore is of central interest, we apply a dynamic Monte Carlo method, ${ }^{41}$ that is known to correspond to the Rouse model. ${ }^{42,43}$ For the present model, an elementary attempted Monte Carlo move is performed by picking an effective monomer at random and trying to displace it from its position $(x, y, z)$ to a new position $\left(x^{\prime}, y^{\prime}, z^{\prime}\right)$, with increments $\Delta x=x^{\prime}-x, \Delta y=y^{\prime}-y, \Delta z=z^{\prime}-z$ chosen randomly from the intervals ${ }^{31}-0.5 \leqslant \Delta x, \Delta y, \Delta z<+0.5$. These trial moves are accepted as new configurations if they pass the standard Metropolis acceptance test. ${ }^{41}$ As has been discussed extensively in the literature, ${ }^{31,32,41,44}$ such Monte Carlo algorithms based on local moves of the monomers realize Rouse model dynamics for the polymer chain. In reality, hydrodynamic interactions by the solvent might give rise to a Zimm model-type ${ }^{43,45}$ dynamics of that part of the chain that is not adsorbed (in the region below the membrane in Fig. 1), but these interactions are ignored in the other treatments throughout $^{8-23}$ and presumably are unimportant on the time scale of the slow translocation process. The local moves of the effective beads in the Monte Carlo sampling can be thought of as conformational changes of the chain where groups of chemical monomers rearrange their configuration by thermally activated transitions over barriers in the torsion potentials. ${ }^{41,44}$ The time evolution of the chain conformation then can be described by a master equation, ${ }^{41,44}$ and thus the simulation is similar in spirit to the analytical models ${ }^{8-16}$ that are based on Langevin and Fokker-Planck-type descriptions of the process.

Finally, we add a comment on the initial condition of our simulations. We are not addressing the full problem, where a dilute solution of chains, which are initially all on the repulsive side of the membrane, in a large volume is considered, and one asks the question how long it takes for one of the chains to pass the pore and get adsorbed on the attractive side of the membrane. Rather we assume that the first stage of the process, where a chain end has entered the pore and got adsorbed on the attractive side has already taken place. In fact, we initialize our system such that $N-N_{\text {cis }}$ monomers are on the "trans" side ${ }^{10}$ of the membrane, where the attractive potential acts, while $N_{c i s}$ monomers are on the repulsive "cis" side, ${ }^{10}$ treating $N_{c i s}$ as an additional parameter. The problem where $N_{c i s}=N-1$ then means that just a chain end has passed the pore. The probability, that this configuration is reached from a simulation where a dilute solution interacts with the repulsive wall, can be related to the partition function of a "polymer mushroom" $Z_{1}(N)$ where a chain "anchors" with one end at the repulsive wall, ${ }^{46-48}$

$$
Z_{1}(N) \propto N^{\gamma_{1}-1} \mu^{N},
$$

while the partition function of a chain in the bulk dilute solution is

$$
Z(N) \propto N^{\gamma_{b}-1} \mu^{N},
$$

$\mu$ being the chemical potential per monomer and $\gamma_{1}, \gamma_{b}$ are the surface and the bulk exponents ${ }^{47,48}$ which in the good solvent case have the values ${ }^{47} \gamma_{1} \approx 0.68 \pm 0.01$ and $^{49} \gamma_{b}$ $=1.1596 \pm 0.0020$ (while for Gaussian chains considered by Park and Sung ${ }^{10}$ these exponents would be ${ }^{44} \gamma_{1}=1 / 2, \gamma_{1}$ $=1$ ). Considering then a volume $V=N n / c$ available on the cis side of the membrane, $n$ being the number of chains, and $c$ being the monomer concentration in the solution, the probability that a chain end enters the pore is proportional to (we here neglected the distinction between $N$ and $N-1$ for large $N)$

$$
p(N) \propto(c / N)\left[Z_{1}(N) / Z(N)\right] \propto c N^{\gamma_{1}-\gamma_{b}-1} \approx c N^{-1.48} .
$$

In the dilute limit $c$ must be very small, and for large $N$ this probability $p(N)$ that a chain end enters the pore is extremely small. Since this probability can be estimated analytically, as outlined above, there would be no point in wasting computer resources for an attempt of estimating this probability by a brute force simulation. This separation of the problem into a problem that a chain end "finds" the gate into the pore, and then the threading of the chain through the pore has also been done in the other studies. The initialization choosing $N_{c i s}, N-N_{c i s}$ is done by joining two polymers of chain lengths $N_{c i s}, N-N_{c i s}$ on the two sides of the membrane at their chain ends, fixing this common chain end at the position in the center of the pore, and equilibrating first the chain configuration under this constraint. Equilibrium is monitored by watching the time evolution of the gyration tensor components of both parts of the chain in the cis and trans region, respectively. Then the clock is set at time $t$ $=0$, and the constraint to fix the monomer inside the pore is removed, so all monomers can move freely.

\section{SIMULATION RESULTS ON THE TRANSLOCATION DYNAMICS}

After having initialized the chain configuration as described in the preceding section, it is in principle straightforward to run the Monte Carlo simulation ${ }^{41}$ and record, e.g., quantities like the fraction of translocated segments as a function of time (Fig. 2). However, it is seen that huge fluctuations occur, and hence we have found it necessary to carry out $M=1000$ individual runs for each choice of parameters. In principle, we thus obtain complete information on the probability distribution $P\left(N_{\text {trans }}, N_{\text {cis }}, N, t\right)$ that at time $t$ after the start with $N-N_{\text {cis }}$ monomers on the trans side the number has evolved from $N-N_{\text {cis }}=N_{\text {trans }}(t=0)$ to $N_{\text {trans }}(t)$. However, due to statistical problems we have not yet attempted a full analysis of this probability distribution, but rather focus only on the average fraction of trans and cis segments

$$
\begin{aligned}
& c_{\text {trans }}(t)=\frac{1}{N} \int N_{\text {trans }} P\left(N_{\text {trans }}, N_{\text {cis }}, N, t\right) d N_{\text {trans }}, \\
& c_{\text {cis }}(t)=1-c_{\text {trans }}(t)
\end{aligned}
$$




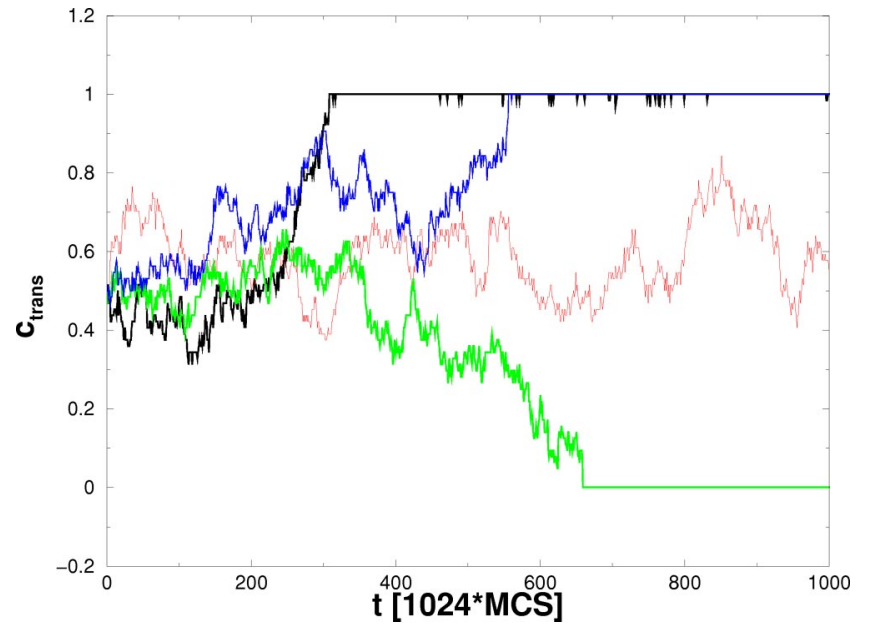

FIG. 2. "Raw Monte Carlo data" for the fraction of translocated segments that have passed the pore from the cis side to the trans side as a function of time (in units of $1024 \mathrm{MCS}$ per bead), for $N=64, \varepsilon=2.2$, and $N_{\text {cis }}=32$.

and we also consider the distribution function of the translocation time $\tau_{\text {trans }}$. This time is well defined for each individual run when the fraction of trans segments becomes unity for the first time (Fig. 2). Note however, that for some choices of $N_{c i s}$ (such as $N_{c i s}=32$ for $N=64$, see Fig. 2) there are runs where this fraction reaches zero rather than unity, i.e., this chain has escaped to the solution on the repulsive side of the membrane, and does not pass the pore at all, although initially a fraction of $50 \%$ of the effective monomers was already on the trans side of the membrane. This observation indicates that for the chosen model translocation involves crossing of a high free energy barrier in phase space, as expected. Figure 2 also shows a run where even after a million Monte Carlo Steps (MCS) it is unclear whether $N_{\text {trans }}$ will settle down at $N$ or go to zero. In principle, the fraction of these "inconclusive" runs could be made as small as desired by simply increasing the length of the runs sufficiently; in practice we have discarded such inconclusive runs from the sampling, to make the statistical effort manageable, and try to correct for this neglect in our estimation of the average translocation time analytically. Each run consists of 2048 "measurements" over time intervals $\Delta t$, whose length was chosen proportional to $N^{2}$, namely, $\Delta t=256(N=32), 1024(N=64), 4096(N=128)$, and $16384(N=256)$, respectively.

Since Fig. 2 shows that ultimately only a part of the chains reach the trans side, one can also ask the question how do $c_{\text {trans }}(t \rightarrow \infty), c_{c i s}(t \rightarrow \infty)$ depend on the initial fraction $c_{c i s}(t=0)=N_{\text {cis }} / N$ of effective monomers that is on the repulsive side of the membrane (Fig. 3). One can see that for short chains, such as $N=32$, there is an appreciable fraction of chains that do not get translocated as soon as $c_{c i s}(0)$ exceeds about $20 \%$, irrespective of $\varepsilon$ (note that $\varepsilon=1.8$ is somewhat below and $\varepsilon=2.2$ is somewhat above the adsorption threshold $\varepsilon_{c} \approx 1.9 \pm 0.05$ of the "mushroom" to "pancake" adsorption transition for this model $\left.{ }^{34}\right)$. However, as the chains become longer [Figs. 3(b)-3(d)], the chances for successful translocation grow rapidly if $c_{c i s}(0)$ is kept constant, provided $\varepsilon>\varepsilon_{c}$. In contrast, the behavior for $\varepsilon<\varepsilon_{c}$ does not depend significantly on $N$ at all, as Fig. 4(a) shows: the curves for $c_{\text {trans }}(\infty)$ for different $N$ completely superimpose, within statistical error! Thus, for this distance from the adsorption transition $c_{c i s}(0)$ must be less than about 0.6, in order that more than $50 \%$ of the chains reach the adsorbing side of the membrane. No such scaling is observed for the fraction of "undecided chains," however: this is expected, of course, since our time cutoff $\left(t_{\max }=2.048\right.$ million MCS for $N=64$, and $t_{\max }$ scales with $N^{2}$ as $\Delta t$ does) turns out to increase with a somewhat smaller power of $N$, as the translocation time does (see below). Due to the need to average over many runs, a choice of $t_{\max }$ much larger than used here would be prohibitively costly, of course, and therefore has not been attempted.

Figure 4(b) reveals a very different behavior for $\varepsilon$ $>\varepsilon_{c}$, however: now the dependence on $N$ is extremely pronounced, and the trend seen suggests that $c_{\text {trans }}(\infty)=1$ for $N \rightarrow \infty$ irrespective of $c_{c i s}(0)$, as long as $c_{c i s}(0)<1$. For very long chains an arbitrarily small fraction of adsorbed monomers suffices as a driving force to pull the chain through the pore!

To analyze this point further, we plot $c_{\text {trans }}(\infty)$ versus $\left[1-c_{c i s}(0)\right] N$ in Fig. 5 : it is seen that now the data converge rather fast to universal curves that no longer depend on $N$. This implies, for a fraction of $1-c_{c i s}(0) \approx 0.1 / N$ already more than $50 \%$ of the chains move through the pore. The reason for this very different size dependence for $\varepsilon<\varepsilon_{c}$ and $\varepsilon>\varepsilon_{c}$ is that for $\varepsilon<\varepsilon_{c}$ only a finite number of monomers is adsorbed at the wall, irrespective of $N$, while for $\varepsilon>\varepsilon_{c}$ a finite fraction of monomers of the chain is adsorbed. Thus, for $\varepsilon>\varepsilon_{c}$ the number of adsorbed sites increases with $N$ \{actually this increase is predicted to be proportional to ${ }^{47}$ $N_{1}=N^{\varphi} f\left[\left(\varepsilon / \varepsilon_{c}-1\right) N^{\varphi}\right] \propto\left(\varepsilon / \varepsilon_{c}-1\right)^{1 / \varphi-1} N$, where the crossover exponent $\varphi$ is ${ }^{50-52} \varphi \approx 0.50 \pm 0.02$, and thus $N_{1}$ $\propto\left(\varepsilon / \varepsilon_{c}-1\right) N$ for $\left.N \rightarrow \infty\right\}$. As a consequence, we conclude that for $\varepsilon>\varepsilon_{c}$ the driving force that pulls the chain through the pore increases linearly with the chain length $N$. On the other hand, the entropic barrier that needs to be overcome threading the chain through the pore varies only logarithmically with $N$. From Fig. 4(a) we conclude that the entropic barrier roughly occurs for $N_{c i s} \approx N / 2$. Since the probability for such a configuration is

$P \propto\left[(N / 2)^{\gamma_{1}-1} \mu^{N / 2}\right]\left[(N / 2)^{\gamma_{1}-1} \mu^{N / 2}\right]=(N / 2)^{2 \gamma_{1}-2} \mu^{N}$,

arguing that the two halfs of the chain (for $\varepsilon<\varepsilon_{c}$ ) can be viewed as two independent polymer mushrooms of chain length $N / 2$, we find that the ratio of probabilities at the barrier and at the considered initial state is

$$
P / P_{1} \propto\left[(N / 2)^{2 \gamma_{1}-2} \mu^{N}\right] /\left[N^{\gamma_{1}-1} \mu^{N}\right] \propto N^{\gamma_{1}-1},
$$

we conclude that the free energy barrier that needs to be overcome is proportional to

$$
\Delta F / k_{B} T \propto \ln \left(P_{1} / P\right)=\left(1-\gamma_{1}\right) \ln N, \quad N \rightarrow \infty .
$$

Of course, Eqs. (9)-(11) do not take into account the effect of the adsorption energy on the free energy barrier, and this approximation can hold at best qualitatively for $\epsilon<\epsilon_{c}$, but cannot be accurate for $\epsilon \geqslant \epsilon_{c}$. For $\epsilon=\epsilon_{c}$ we expect that 

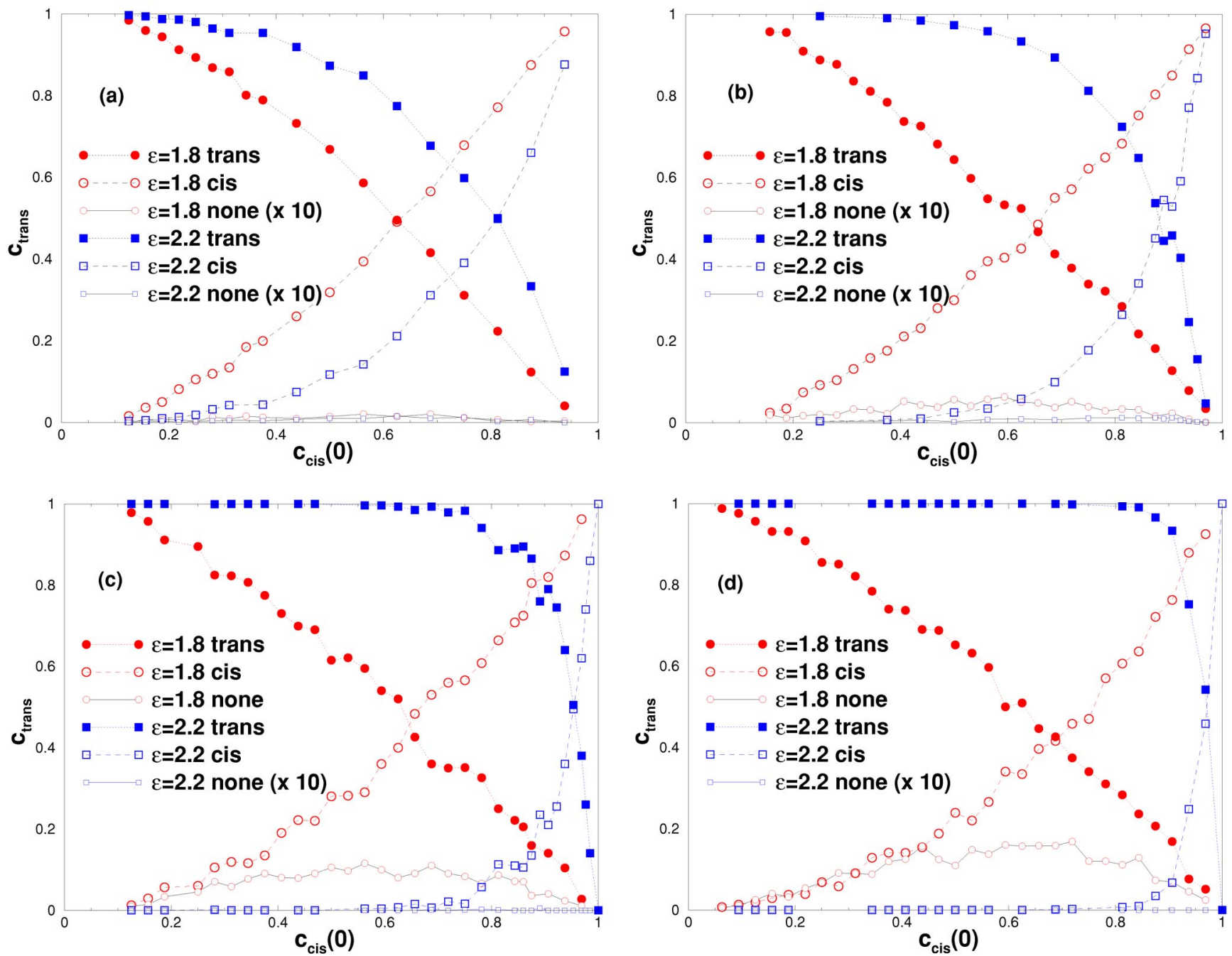

FIG. 3. Plot of $c_{\text {trans }}(\infty)$ and $c_{c i s}(\infty)$ vs $c_{c i s}(0)$ for $N=32$ (a), $N=64$ (b), $N=128$ (c), and $N=256$ (d), showing two choices of $\varepsilon$ as indicated. Note that time $t=\infty$ here actually means $2048 \Delta t$ MCS, with $\Delta t(N=32)=256, \Delta t(N=64)=1024, \Delta t(N=128)=4096$, and $\Delta t(N=256)=16384$; therefore $c_{\text {trans }}(\infty)$ $+c_{c i s}(\infty)=1-c_{\text {none }}$, with $c_{\text {none }}$ being the fraction of chains which have not yet reached either $N_{\text {trans }}=N$ or $N_{\text {trans }}=0$ during that time. This fraction $c_{\text {none }}$ of "undecided chains" is also included in the figure (magnified by a factor 10 for clarity), to illustrate the possible errors in our analysis.
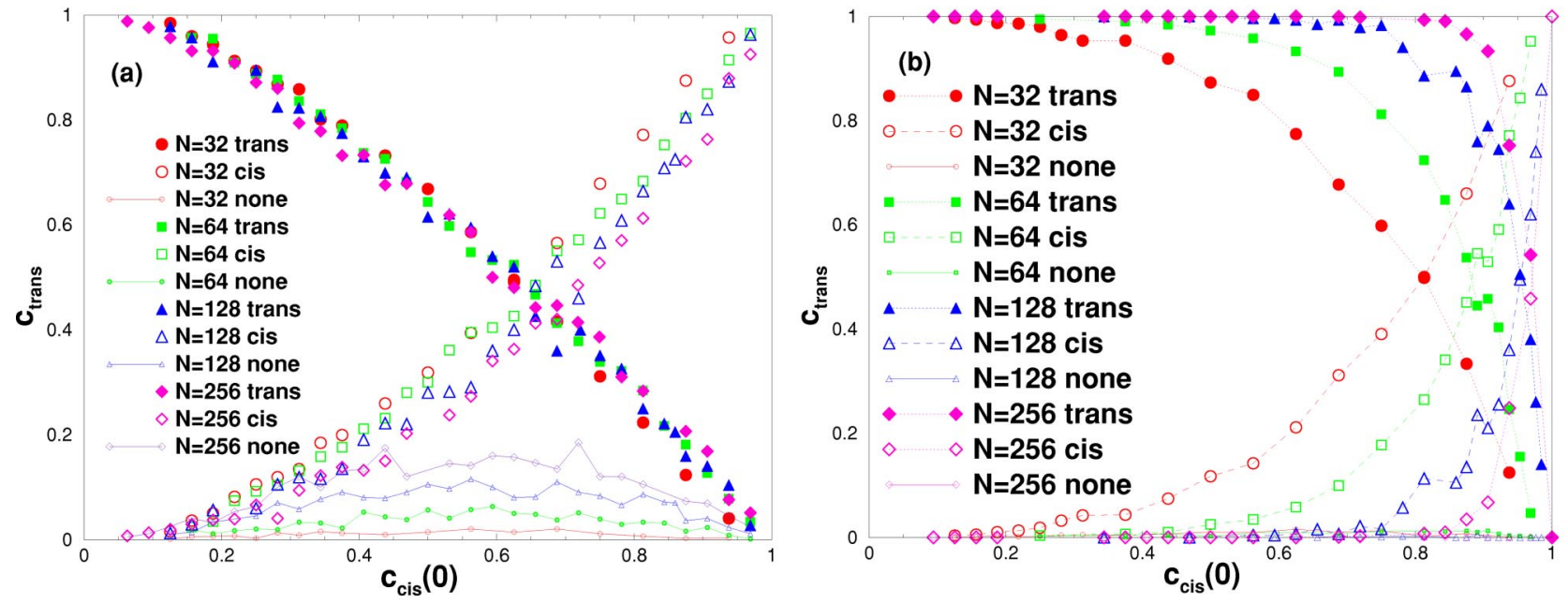

FIG. 4. Plot of $c_{\text {trans }}(\infty)$ and $c_{c i s}(\infty)$ vs $c_{c i s}(0)$ for four different choices of $N$, as indicated; the fraction $c_{\text {none }}$ of undecided chains is also included. Case (a) shows the choice $\varepsilon=1.8$, case (b) shows the choice $\varepsilon=2.2$. 


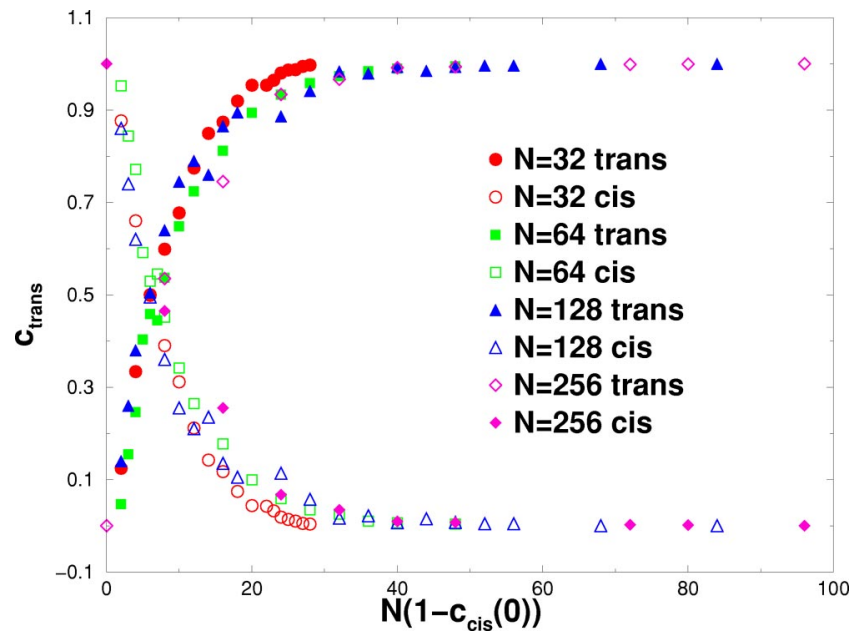

FIG. 5. Plot of $c_{\text {trans }}(\infty)$ and $c_{c i s}(\infty)$ vs $\left[1-c_{c i s}(0)\right] N$ for $\varepsilon=2.2$ and four choices of $N$.

the probability of a configuration where $N_{\text {cis }}$ monomers are on the repulsive side and $N-N_{c i s}$ monomers are on the attractive side of the pore is given by

$$
\begin{aligned}
P \propto & {\left[N_{c i s}^{\gamma_{1}-1} \mu^{N_{c i s}}\left(N-N_{c i s}\right)^{\gamma_{1 m}-1} \mu^{N-N_{c i s}}\right] } \\
& =N_{c i s}^{\gamma_{1}-1}\left(N-N_{c i s}\right)^{\gamma_{1 m}-1} \mu^{N},
\end{aligned}
$$

where $\gamma_{1 \mathrm{~m}}$ is the surface-bulk multicritical exponent (cf., Refs. 47 and 49). Since $\gamma_{1 m}>\gamma_{1}$, the free energy barrier (corresponding to the minimum of $P$ ) then no longer corresponds to $N_{c i s}=N / 2$, but to a larger value of $N_{c i s}$. For $\epsilon$ $>\epsilon_{c}$ the analogous result is

$$
P \propto\left[N_{c i s}^{\gamma_{1}-1} \mu^{N_{c i s}}\right]\left[\left(N-N_{c i s}\right)^{\gamma^{2 d}-1} \mu_{a d}^{N-N_{c i s}}\right],
$$

where $\gamma^{2 d}$ is the critical exponent for the number of configurations of two-dimensional self-avoiding walks, and $\mu_{a d}$ contains a term taking into account the adsorption energy. As a result, the barrier occurs for $N_{\text {trans }}=N-N_{\text {cis }} \ll N$ if $\epsilon$ $>\epsilon_{c}$, rather than for $N_{\text {trans }}=N_{\text {cis }}=N / 2$. Unfortunately, for our choice of $N$ and $\epsilon$ these asymptotic laws presumably no longer hold.

Inspection of Fig. 5 suggests that the "dividing line" where $c_{\text {trans }}(\infty)=0.5$ corresponding to the top of the barrier means that about seven monomers need to be adsorbed on the trans side for $\varepsilon=2.2$.

We now turn to a study of the translocation times. From Fig. 2 we note that for each individual run it is a well-defined question to record the time when the fraction of monomers $c_{\text {trans }}(t)$ becomes equal to unity (for the first time in this particular run). In this way it is straightforward to record a distribution function of translocation times (Fig. 6). Also a fit to the form $P(\tau) \propto \tau^{3 / 2} \exp \left(-\tau / \tau_{\text {trans }}\right)$ is included; note that similar distributions were obtained occasionally from the analytical theories. ${ }^{12}$ However, it is apparent that there are systematic deviations of the data from this ad hoc formula, particularly at small $\tau$, but the accuracy of the numerical data does not allow a unique determination of the functional form of $P(\tau)$. Even larger scatter $P(\tau)$ was found for $\varepsilon<\varepsilon_{c}$, however, and hence no attempt is made to analyze those data here.

Figure 7 presents a log-log plot of the resulting average translocation time $\tau_{\text {trans }}$ versus the fraction $c_{\text {cis }}(t=0)$. When $c_{c i s}(t=0) \geqslant 0.5$, a saturation of the time is reached, particularly for $\varepsilon<\varepsilon_{c}$. It also is obvious that for large $N$ the translocation times for $\varepsilon<\varepsilon_{c}$ are much larger than those for $\varepsilon>\varepsilon_{c}$, while for not so long $N$ there is not much difference. However, the most interesting result clearly is the apparent power law behavior for $c_{c i s}(t=0) \ll 1$

$$
\tau_{\text {trans }} \propto\left[c_{\text {cis }}(t=0)\right]^{\varsigma}, \quad \varsigma=1.30 \pm 0.01 .
$$

In this regime where $c_{c i s}(t=0) \ll 1$ the force which drags the part of the chain that has remained on the cis side of the membrane is essentially independent of $c_{c i s}(t=0)$. The center of mass of this part at time $t$ is at a position of order $\left[N c_{c i s}(t)\right]^{\nu}$. Since the force acting on the monomer that is in the pore must pull the center of mass of the cis part over the quoted distance one does expect that $\tau_{\text {trans }}$ should
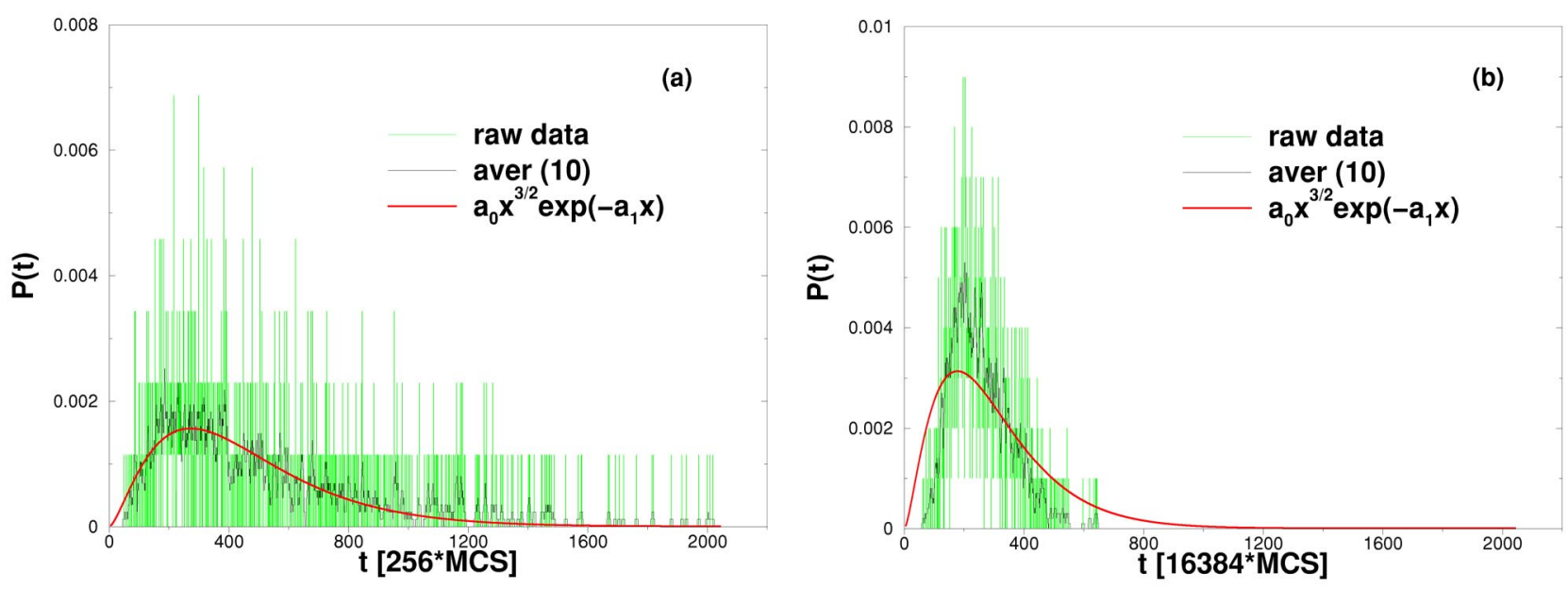

FIG. 6. Distribution function $P(t)$ of translocation times $t$ for $\varepsilon=2.2, N=32, N_{c i s}=16$ (a) and $\varepsilon=2.2, N=256, N_{c i s}=128$ (b). Both raw data and an average over $\delta t=10$ neighboring entries are shown, and a fit of the form $a_{0} x^{3 / 2} \exp \left(-a_{1} x\right)$ is included. 


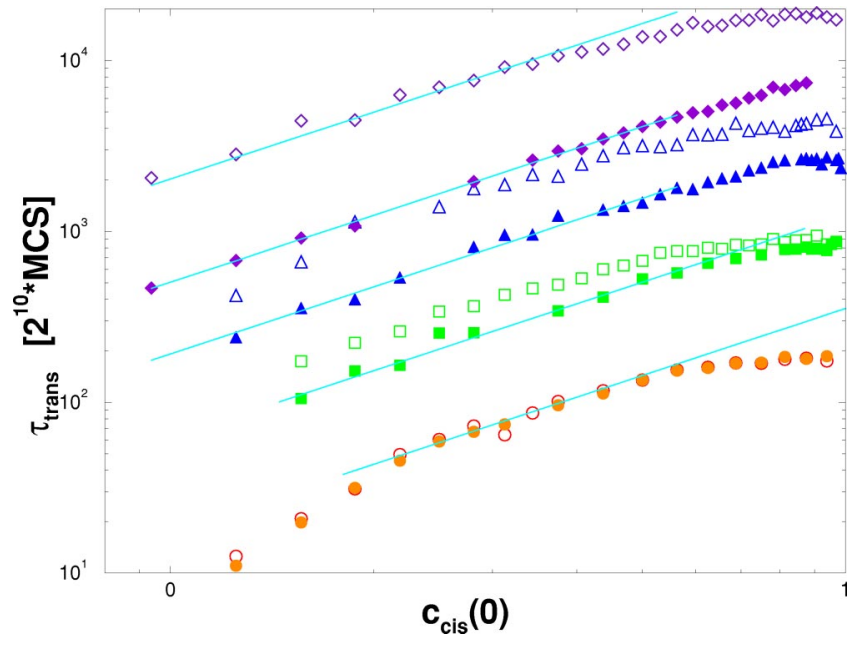

FIG. 7. Log-log plot of the translocation time $\tau_{\text {trans }}$ vs the fraction $c_{c i s}(t$ $=0$ ) of monomers that are initially on the repulsive side of the membrane. Several choices of $N$ are shown in $(\bigcirc-N=32, \square-N=64, \triangle-N=128, \diamond$ $-N=256$ ), both for $\varepsilon=1.8$ (empty symbols) and $\varepsilon=2.2$ (full symbols). Straight lines indicate the power law, Eq. (12).

increase with a power of $c_{c i s}$ that is at least as large as $\nu$ $\approx 0.59$, but clearly a more elaborate treatment is needed to explain the exponent $s$ in Eq. (12).

Finally we turn to the chain length dependence of the translocation time. Figure 8 indicates again power law behavior, which we write in terms of the dynamic exponent $z$ ( $R_{g}$ being the gyration radius of a free chain)

$$
\begin{aligned}
& \tau_{\text {trans }} \propto R_{g}^{z} \propto N^{z \nu}, \quad z \nu=2.23 \pm 0.04\left(\varepsilon<\varepsilon_{c}\right) \\
& \text { and } z \nu=1.65 \pm 0.08\left(\varepsilon>\varepsilon_{c}\right) .
\end{aligned}
$$

Unfortunately, these estimates for the exponent $z$ are in disagreement with the corresponding literature; Park and Sung $^{10}$ suggested $z \nu=3\left(\varepsilon<\varepsilon_{c}\right)$ and $z \nu=2\left(\varepsilon>\varepsilon_{c}\right)$, while we find significantly smaller values. We shall discuss our findings in more detail in the following section.

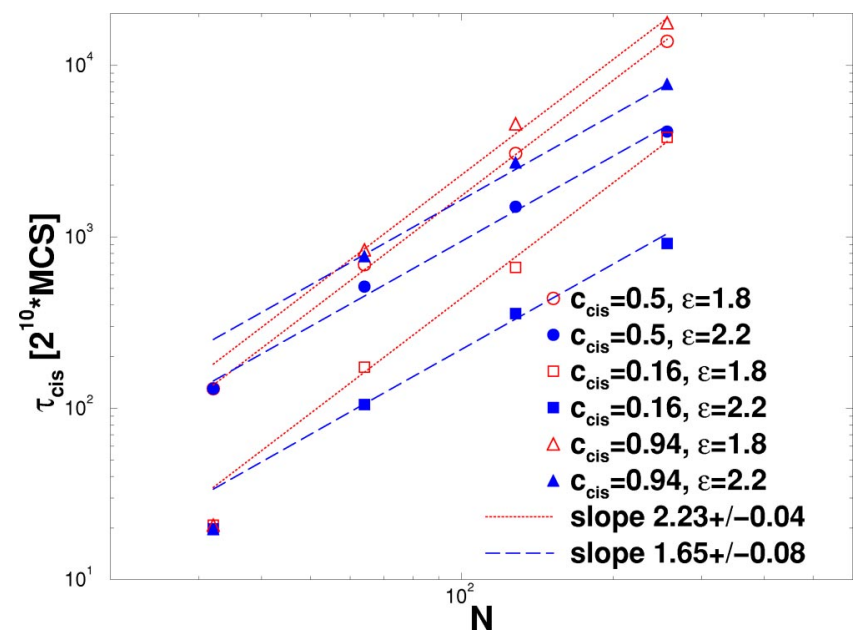

FIG. 8. Log-log plot of the translocation time $\tau_{\text {trans }}$ vs chain length $N$. Open symbols refer to $\varepsilon=1.8$, full symbols refer to $\varepsilon=2.2$. Several choices of $c_{\text {cis }}$ are shown, as indicated in the figure. Straight lines indicate possible power law fits.

\section{DISCUSSION: TO WHAT EXTENT IS THE SCALING OF THE TRANSLOCATION TIME WITH CHAIN LENGTH UNDERSTOOD?}

In the preceding section, we have already noted the disagreement of our results for the dynamic exponent $z$ of the translocation time with the estimates of Park and Sung. ${ }^{10}$ In order to understand the reasons for this discrepancy, let us recall the essential point of their derivation. ${ }^{10}$ In the special case $\varepsilon=0$, when both sides of the membrane are repulsive, the problem reduces to the threading of a chain through a hole in a membrane, by free diffusive motion., ${ }^{9,11,13,22}$ Park and Sung ${ }^{9}$ suggest that one can simply consider the problem as equivalent to the diffusion of a particle with coordinate $N_{\text {trans }}(t)$ from $N_{\text {trans }}(0)=1$ to $N_{\text {trans }}(t \rightarrow \infty)=N$ over a potential barrier

$$
\Delta S / k_{B}=\left(1-\gamma_{1}\right)\left[\ln N_{c i s}+\ln \left(N-N_{c i s}\right)-\ln N\right] .
$$

Note that $\Delta S / k_{B}=0$ if $N_{c i s}=1$ or if $N-N_{\text {cis }}=1$, provided $N$ is large enough such that $\ln (N-1) \approx \ln N$. The maximum of this barrier occurs for $N_{c i s}=N-N_{c i s}=N / 2$, and this is the result $\Delta S^{\max } / k_{B}=\left(1-\gamma_{1}\right)[\ln N-2 \ln 2] \approx\left(1-\gamma_{1}\right) \ln N$ already anticipated in Eq. (11). Assuming then that at each stage of the translocation process the chain is in local equilibrium, so Eq. (14) acts as a driving force for the diffusive motion of $N_{\text {trans }}(t)$, one finds

$$
\tau_{\text {trans }} \propto N^{2} / \mathcal{D}
$$

irrespective of whether or not excluded volume interactions are present. However, it remains to identify the meaning of the diffusion constant $\mathcal{D}$ in Eq. (15). Sung and Park ${ }^{9,10}$ suggested that $\mathcal{D}$ should be identified with the diffusion constant of a free chain, which in the Rouse model scales as $\mathcal{D}$ $\propto W \ell^{2} / N$, where $W$ is the jump rate of a monomeric unit and $\ell$ a characteristic jump distance. As a result Sung and Park ${ }^{9}$ predicted $\tau_{\text {trans }} \propto N^{3}$. As noted by them, this is the same power law as for a reptating chain in a dense melt! Clearly, this result is highly implausible, because a pore in a very thin membrane acts like the cross section of a tube in the reptation problem only in a single point along the primitive path of the chain, while otherwise the chain parts on the two sides of the membrane can execute a fully free Rouse-like motion. From the Monte Carlo study of mushrooms and adsorbed chains, ${ }^{34}$ there is clear evidence that chains interacting with a surface have a relaxation time of the same order as in the bulk $\left(\tau_{\text {Rouse }} \propto N^{2 \nu+1}\right.$ for the Rouse model, ${ }^{30}$ where $\nu \approx 0.59$ in $d=3$ dimensions while $\nu=3 / 4$ for strongly adsorbed chains in two-dimensional geometry ${ }^{34}$ ). Consequently, Muthukumar $^{11}$ suggested that $\mathcal{D}$ in Eq. (15) is not the diffusion constant of a whole chain, but rather the diffusion constant of the monomer that just passes the hole, and hence a constant, independent of $N$. While some simulations using rather short chains ${ }^{22}$ seem to support the results of Muthukumar, Chuang et al. ${ }^{13}$ vividly criticized this approach: in fact, Muthukumar's ${ }^{11}$ result implies that a chain on top of the barrier $\left(N_{\text {cis }}=N_{\text {trans }}=N / 2\right)$, which does not experience any driving force whatsoever, has a translocation time $\tau_{\text {trans }}$ $\propto N^{2}$, which is less than the relaxation time of an unconstrained free chain, $\tau_{\text {Rouse }}$. It is clear that $\tau_{\text {Rouse }}$ must be a lower bound to the translocation time, and hence the result of 
Muthukumar clearly is invalid. Chuang et al. ${ }^{13}$ identify the flaw in the model of Brownian motion over the barrier, Eq. (14): due to the correlations between the monomer in the pore and the remaining monomers of the polymer in the halfspaces away from the membrane, the monomer number $N_{\text {trans }}(t)-1$ in the pore does not execute a simple diffusive motion, rather anomalous diffusion along the coordinate system labeling the monomers along the chain occurs. As a matter of fact, the relaxation time $\tau_{\text {Rouse }} \propto N^{2 \nu+1}$ exceeds the predicted translocation time, which is based on the idea that there is enough time to equilibrate the cis and trans parts of the chain, while the fact that $\tau_{\text {Rouse }}$ would exceed $\tau_{\text {trans }}$ shows that the treatment lacks self-consistency. Chuang et al. $^{13}$ argue, on the basis of simulations in $d=2$ dimensions, that the actual translocation time scales with the same exponent as the Rouse time, only the prefactor is distinctly larger. In fact, in $d=3$ dimensions their analysis would imply that

$$
\tau_{\text {trans }} \propto \tau_{\text {Rouse }} \propto N^{2 \nu+1}=N^{2.18},
$$

and this result indeed is compatible with our findings for $\varepsilon$ $<\varepsilon_{c}\{$ Eq. (13)\}. One should note that Eq. (16) holds for $\varepsilon$ $<\varepsilon_{c}$ and for cases where a finite fraction of the monomers of the chain has already passed the hole from the cis to the trans side, cf. Fig. 4(a), and one asks the question how long does it take for the chains that fully pass the hole to get fully translocated. However, Fig. 4 shows that in the limit where $c_{c i s}(0) \rightarrow 1$ the fraction of translocated chains vanishes linearly,

$$
N_{\text {trans }}(\infty) / N \propto 1-c_{\text {cis }}(0) .
$$

Thus the probability that a chain which has just entered the pore with one chain end gets translocated at all is small and is of order $1 / N$. This low probability reflects the free energy barrier, Eq. (14), that is difficult to cross. If one asks the question how long does it take on average that a chain is translocated, one has to take this low translocation probability into account: only of the order 1 out of $N$ chains that get their chain end into the pore actually get through the pore, but those few chains that diffuse through need a time $\tau_{\text {trans }}$ as quoted in Eq. (16).

Figures 4 and 5 imply that for $N \rightarrow \infty$ this problem of polymer translocation is a kind of first-order phase transition, in the sense as described by diMarzio and Mandell ${ }^{9}$ for translocation driven by a chemical potential difference: for $N$ $\rightarrow \infty$ and $\varepsilon<\varepsilon_{c}$, we have $N_{\text {trans }}(\infty) / N=0$ for $c_{c i s}(0) \rightarrow 1$, while for $N \rightarrow \infty$ and $\varepsilon>\varepsilon_{c}$, we have $N_{\text {trans }}(\infty) / N=1$ if we take the limit $c_{c i s}(0) \rightarrow 1$ after the limit $N \rightarrow \infty$.

We now discuss the behavior of the time in the presence of a driving force.

Chuang et al. ${ }^{13}$ suggest for the pulling velocity of the translocation coordinate a scaling relation, $\Delta \mu$ being the chemical potential difference driving the translocation, $\widetilde{v}$ being a scaling function depending on the ratio of the driving energy and the thermal energy,

$$
\frac{d N_{\text {trans }}(t)}{d t} \propto \frac{N}{\tau_{\text {Rouse }}} \widetilde{v}\left(\frac{\Delta \mu N}{k_{B} T}\right) \propto N^{2-z \nu} \Delta \mu, \quad N \rightarrow \infty .
$$

From Eq. (16) one hence concludes that for $\Delta \mu>0$ the translocation time [needed for $N_{\text {trans }}(t)=1$ to develop towards $N_{\text {trans }}(t)=N$, using Eq. (16)] is of the order $\tau_{\text {trans }}(\Delta \mu>0) \propto N^{z \nu-1} \approx N^{1.18}$. This relation replaces the result of Muthukumar ${ }^{11} \tau_{\text {trans }}(\Delta \mu>0) \propto N$, and presumably is also compatible with the existing simulations. ${ }^{22}$

We now wish to generalize Eq. (16) to the case of an adsorbing wall.

Here we note that during the translocation process the energy won by the adsorption of the translocating chain is proportional to $\left(\varepsilon-\varepsilon_{c}\right) N_{\text {trans }}(t)$ for $\varepsilon>\varepsilon_{c}$ and proportional to $N_{\text {trans }}^{\varphi}(t)$ for $\varepsilon=\varepsilon_{c}, \varphi$ being the crossover exponent ${ }^{50-52}$ mentioned above. This energy replaces the energy $\Delta \mu N$ in Eq. (16), and thus,

$$
\begin{aligned}
\frac{d N_{\text {trans }}(t)}{d t} & \propto \frac{N}{\tau_{\text {Rouse }}} \widetilde{v}\left[\text { const } N_{\text {trans }}^{\varphi}(t)\right] \\
& \propto \frac{N}{\tau_{\text {Rouse }}}\left[N_{\text {trans }}(t)\right]^{\varphi}, \quad \varepsilon=\varepsilon_{c},
\end{aligned}
$$

which yields

$$
N_{\text {trans }}^{-\varphi} d N_{\text {trans }} \propto N d t / \tau_{\text {Rouse }},
$$

and integrating this relation from $t=0$ to $t=\tau_{\text {trans }}$ (i.e., from $N_{\text {trans }}=1$ to $N_{\text {trans }}=N$ ) yields, using Eq. (16)

$$
\tau_{\text {trans }}\left(\varepsilon=\varepsilon_{c}\right)=\tau_{\text {Rouse }} N^{-\phi} \propto N^{1.68} .
$$

Similarly, for $\varepsilon>\varepsilon_{c}$ we have

$$
\begin{aligned}
\frac{d N_{\text {trans }}(t)}{d t} & \propto \frac{N}{\tau_{\text {Rouse }}} \widetilde{v}\left[\frac{\varepsilon-\varepsilon_{c}}{k_{B} T} N_{\text {trans }}(t)\right] \\
& \propto \frac{N}{\tau_{\text {Rouse }}} \frac{\varepsilon-\varepsilon_{c}}{k_{B} T} N_{\text {trans }}(t),
\end{aligned}
$$

and hence

$$
N_{\text {trans }}^{-1} d N_{\text {trans }} \propto N \frac{\varepsilon-\varepsilon_{c}}{k_{B} T} d t / \tau_{\text {Rouse }},
$$

which is integrated to give

$$
\tau_{\text {trans }}\left(\varepsilon>\varepsilon_{c}\right) \propto \tau_{\text {Rouse }} \frac{k_{B} T}{\varepsilon-\varepsilon_{c}} \frac{\ln N}{N} \propto N^{1.18} \ln N .
$$

Since for $32 \leqslant N \leqslant 256$ the function $\ln N$ is similar to $N^{0.2}$, the "effective exponent" resulting from Eq. (24) is $\tau_{\text {trans }} \propto N^{1.4}$. For the numerical results of the preceding section (Fig. 8), we expect to be in a crossover regime between Eqs. (21) and (24), and this conjecture is in fact compatible with the numerical data.

Finally we comment on the translocation time that is found when the initial condition is $N_{\text {cis }}(t=0) / N=c_{\text {cis }}(0)$ $\ll 1$, so most of the monomers at the start of the simulation already are on the trans side. These monomers act as a driving force on the remaining ones, and hence a phenomenological ansatz similar in spirit to Eq. (22) is

$\frac{d N_{\text {trans }}(t)}{d t} \propto \frac{N}{\tau_{\text {Rouse }}} N_{\text {trans }}(t), \quad N_{\text {trans }}^{-1} d N_{\text {trans }} \propto \frac{N}{\tau_{\text {trans }}} d t$,

and hence 


$$
\begin{aligned}
& \tau_{\text {trans }} \propto\left\{\ln N-\ln \left[N-N_{\text {cis }}(0)\right]\right\} \tau_{\text {Rouse }} / N \\
&=-\tau_{\text {Rouse }} \frac{\ln \left[1-c_{\text {cis }}(0)\right]}{N}
\end{aligned}
$$

For small $c_{c i s}(0) \ll 1$ this result would imply $\tau_{\text {trans }}$ $\propto c_{\text {cis }}(0)$ rather than $\tau_{\text {trans }} \propto\left[c_{\text {cis }}(0)\right]^{1.3}$ seen in Fig. 7. Unfortunately, we do not see an explanation for this discrepancy.

\section{CONCLUSIONS}

In this investigation, Monte Carlo results for the translocation of flexible polymers through pores in membranes were presented, assuming that the driving force for the process is an asymmetry in the monomer-membrane interactions, the latter being purely repulsive on the cis side, but attractive (and in the vicinity of a mushroom to pancake transition of an endgrafted polymer) on the trans side of the membrane. We have assumed good solvent conditions in the solution on both sides of the membrane, i.e., we include excluded volume interactions among the monomers, but ignore hydrodynamic forces (the dynamic Monte Carlo method of our coarse-gained model hence results in a Rouse-like relaxation). We restrict attention to the case where the membrane is very thin (thickness of the order of the size of an effective monomer) and the pore is very narrow (containing a single monomer at any time of the translocation process), to make contact with the theoretical models of Sung and Park $^{9,10}$ and Muthukumar. ${ }^{11}$ We vary the chain length of our bead-spring model for about one decade $(32 \leqslant N \leqslant 256)$ and typically average over 1000 runs, choosing the number $N_{c i s}(0)$ of monomers that initially are on the repulsive cis side of the membrane, as an additional parameter. We focus on the scaling behavior of the translocation time $\tau$ and its distribution.

Below the adsorption threshold $\left(\varepsilon<\varepsilon_{c} \approx 1.9\right.$ in our model) we find that $\tau \propto N^{2.23 \pm 0.04}$, consistent with the Rouse model scaling $\tau \propto N^{2 \nu+1}=N^{2.18}$. This result confirms the conclusions of Chuang, Kantor, and Kardar, ${ }^{13}$ and is at variance with the suggestion of Park and Sung ${ }^{10}$ that $\tau \propto N^{3}$ and the result of Muthukumar ${ }^{11}$ that $\tau \propto N^{2}$. Clearly, $\tau \propto N^{3}$ would imply that a single pore in a thin membrane is as severe an obstacle to polymer motion as many entanglements in a dense polymer melt, which is not plausible; on the other hand, $\tau \propto N^{2}$ would imply that the translocating chain relaxes faster (in the presence of excluded volume) than an unconstrained chain in dilute solution, which also is not plausible. ${ }^{13}$ While some simulations have claimed to confirm the result of Muthukumar, ${ }^{11}$ they either considered short chains $^{22}$ or gaussian chains, ${ }^{19}$ for which $2 \nu+1=2$, and hence there is no contradiction with our results.

For $\varepsilon>\varepsilon_{c}$ we obtain $\tau_{\text {trans }} \propto N^{1.65 \pm 0.08}$. Within the statistical errors, this result is in agreement with a new scaling prediction $\tau_{\text {trans }} \propto N^{2 \nu+1-\varphi}$ derived by us for the adsorption threshold $\varepsilon_{c}(\varphi=0.50 \pm 0.02$ being the "crossover exponent").

In addition, interesting results on the fraction of translocating chains were obtained. For $\varepsilon<\varepsilon_{c}$ we found that the fraction of translocating chains varies linearly with
$N_{c i s}(0) / N$, i.e., for $N_{c i s}(0)=1$ (a single monomer has entered the pore) the translocation probability scales like $1 / N$. On the other hand, for $\varepsilon>\varepsilon_{c}$ the translocation probability is of order 1 when $N_{\text {cis }}(0)$ exceeds a finite threshold [of order $N_{\text {cis }}(0)=7$ for $\varepsilon=2.2$ in our model]. Thus, the translocation probability is zero for $\varepsilon<\varepsilon_{c}$ in the limit $N \rightarrow \infty$, but unity for $N \rightarrow \infty$ and $\varepsilon>\varepsilon_{c}$, as soon as a finite fraction of monomers is on the adsorbing side. Thus, the translocation probability is an order parameter of a first-order transition in the sense discussed by diMarzio and Mandell. ${ }^{9}$

A result still waiting for a theoretical explanation is our finding that $\tau_{\text {trans }} \propto\left[N_{\text {cis }}(0) / N\right]^{1.3}$. Also, the precise dependence of $\tau_{\text {trans }}$ on the variable $\varepsilon-\varepsilon_{c}$ still needs to be investigated, as well as the dependence on membrane thickness, pore diameter, chain stiffness, solvent quality, etc. Thus, our study clearly is a first step only, but it confirms the conclusion of Chuang et al. ${ }^{13}$ that the description of the translocation dynamics in terms of a one-dimensional Fokker-Planck equation is too simplistic. We hope to report on some extensions of the present work to clarify the dependence on the parameters mentioned above in the future.

\section{ACKNOWLEDGMENTS}

This work has been supported in part by the Deutsche Forschungs-gemeinschaft (DFG) under Grant Nos. 436 BUL 113/130/1 and SFB 625/A3. Authors are grateful to G.D. Smith for stimulating discussions. A.B. acknowledges partial support from NSF-NIRT grant, local hospitality from the Institute of Physics, Mainz, and discussion with Amit Meller.

${ }^{1}$ J. J. Kasianowicz, E. Brandin, D. Branton, and D. W. Deaner, Proc. Natl. Acad. Sci. U.S.A. 93, 13770 (1996).

${ }^{2}$ M. Aktson, D. Branton, J. J. Kasianowicz, E. Brandin, and D. W. Deaner, Biophys. J. 77, 3227 (1999).

${ }^{3}$ A. Meller, L. Nivon, E. Brandin, J. A. Golovchenko, and D. Branton, Proc. Natl. Acad. Sci. U.S.A. 97, 1079 (2000).

${ }^{4}$ S. E. Henrickson, M. Misakian, B. Robertson, and J. J. Kasianowicz, Phys. Rev. Lett. 85, 3057 (2000).

${ }^{5}$ A. Meller, L. Nivon, and D. Branton, Phys. Rev. Lett. 86, 3435 (2001).

${ }^{6}$ A. F. Sauer-Budge, J. A. Nyamwanda, D. K. Lubensky, and D. Branton, Phys. Rev. Lett. 90, 238101 (2003).

${ }^{7}$ A. Meller, J. Phys.: Condens. Matter 15, R581 (2003).

${ }^{8}$ S. M. Simon, C. S. Reskin, and G. F. Oster, Proc. Natl. Acad. Sci. U.S.A. 89, 3770 (1992).

${ }^{9}$ E. A. diMarzio and A. L. Mandell, J. Chem. Phys. 107, 5510 (1997).

${ }^{10}$ P. J. Park and W. Sung, J. Chem. Phys. 108, 3013 (1998).

${ }^{11}$ M. Muthukumar, J. Chem. Phys. 111, 10371 (1999).

${ }^{12}$ D. K. Lubensky and D. R. Nelson, Biophys. J. 77, 1824 (1999).

${ }^{13}$ J. Chuang, Y. Kantor, and M. Kardar, Phys. Rev. E 65, 011802 (2001).

${ }^{14}$ E. Slonkina and A. B. Kolomeisky, J. Chem. Phys. 118, 7112 (2003); T. Ambjörnsson, S. P. Apell, Z. Konkoli, E. A. DiMarzio, and J. J. Kasianowicz, ibid. 117, 4063 (2002).

${ }^{15}$ U. Gerland, R. Bundschuh, and T. Hwa, Phys. Biol. 1, 19 (2004).

${ }^{16}$ O. Flomenbom and J. Klafter, preprint (cond-mat/0308199).

${ }^{17}$ A. Baumgärtner and J. Skolnick, Phys. Rev. Lett. 74, 2142 (1995).

${ }^{18}$ G. W. Slater, H. L. Guo, and G. I. Nixon, Phys. Rev. Lett. 78, 1170 (1997).

${ }^{19}$ S.-S. Chern, A. E. Cardenas, and R. D. Coalson, J. Chem. Phys. 115, 7772 (2001).

${ }^{20}$ M. Muthukumar, Phys. Rev. Lett. 86, 3188 (2001).

${ }^{21}$ C. Y. Kong and M. Muthukumar, Electrophoresis 23, 2697 (2002); Z. Farkas, I. Derenyi, and T. Vicsek, J. Phys.: Condens. Matter 15, S1767 (2003).

${ }^{22}$ P. Tian and G. D. Smith, J. Chem. Phys. 119, 11475 (2003).

${ }^{23}$ R. Zandi, D. Reguera, J. Rudnick, and W. M. Gelbart, Proc. Natl. Acad. Sci. U.S.A. 100, 8649 (2003). 
${ }^{24}$ B. Alberts and D. Bray, Molecular Biology of the Cell (Garland, New York, 1994).

${ }^{25}$ J. Darnell, H. Lodish, and D. Baltimore, Molecular Cell Biology (Scientific American Books, New York, 1995).

${ }^{26}$ B. Alberts, D. Bray, A. Johnson, J. Lewis, M. Raff, K. Robert, P. Walter, and K. Roberts, Essential Cell Biology (Garland, New York, 1998).

${ }^{27}$ J. Han, S. W. Turner, and H. G. Craighead, Phys. Rev. Lett. 83, 1688 (1999).

${ }^{28}$ S. W. P. Turner, M. Calodi, and H. G. Craighead, Phys. Rev. Lett. 88, 128103 (2002)

${ }^{29}$ D.-C. Chang, Guide to Electroporation and Electrofusion (Academic, New York, 1992).

${ }^{30}$ P. G. de Gennes, Scaling Concepts in Polymer Physics (Cornell University Press, Ithaca, 1979).

${ }^{31}$ I. Gerroff, A. Milchev, W. Paul, and K. Binder, J. Chem. Phys. 98, 6526 (1993).

${ }^{32}$ A. Milchev, W. Paul, and K. Binder, J. Chem. Phys. 99, 4786 (1993).

${ }^{33}$ A. Milchev and K. Binder, Macromol. Theory Simul. 3, 915 (1994).

${ }^{34}$ A. Milchev and K. Binder, Macromolecules 29, 343 (1996).

${ }^{35}$ A. Milchev and K. Binder, J. Physique II (Paris) 6, 21 (1996); Eur. Phys. J. B 9, 477 (1998).

${ }^{36}$ A. Milchev and K. Binder, J. Chem. Phys. 106, 1978 (1997); ibid. 114, 8610 (2001); ibid. 116, 7691 (2002); ibid. 117, 6852 (2002).
${ }^{37}$ K. Kremer and K. Binder, J. Chem. Phys. 81, 6381 (1984).

${ }^{38}$ A. Milchev and K. Binder, Macromol. Theory Simul. 3, 305 (1994).

${ }^{39}$ M. Daoud and P. G. de Gennes, J. Phys. (Paris) 38, 85 (1977).

${ }^{40}$ P. G. de Gennes, Adv. Polym. Sci. 138, 91 (1999).

${ }^{41}$ Monte Carlo and Molecular Dynamics Simulations in Polymer Science, edited by K. Binder (Oxford University Press, New York, 1995).

${ }^{42}$ P. E. Rouse, J. Chem. Phys. 21, 127 (1953).

${ }^{43}$ M. Doi and S. F. Edwards, The Theory of Polymer Dynamics (Clarendon, Oxford, 1986)

${ }^{44}$ K. Binder and W. Paul, J. Polym. Sci., Part B: Polymer Phys. Ed. 35, 1 (1997).

${ }^{45}$ B. Zimm, J. Chem. Phys. 24, 269 (1956).

${ }^{46}$ P. G. de Gennes, J. Phys. (Paris) 37, 1445 (1976); Macromolecules 13 , 1069 (1980).

${ }^{47}$ E. Eisenriegler, K. Kremer, and K. Binder, J. Chem. Phys. 77, 6296 (1982).

${ }^{48}$ E. Eisenriegler, Polymers Near Surfaces (World Scientific, Singapore, 1993).

${ }^{49}$ R. Guida and J. Zinn-Justin, J. Phys. A 31, 8103 (1998).

${ }^{50}$ R. Hegger and P. Grassberger, J. Phys. A 27, 4069 (1994).

${ }^{51}$ H. W. Diehl and M. Shpot, Nucl. Phys. B 528, 595 (1998).

${ }^{52}$ S. Metzger, M. Müller, K. Binder, and J. Baschnagel, Macromol. Theory Simul. 11, 985 (2002). 